\title{
Nível de atividade física e comportamento sedentário de estudantes de educação
}

\author{
física \\ Physical Activity level and sedentary behavior of physical education students \\ Nivel de actividad física y comportamiento sedentario de estudiantes de educación física
}

Recebido: 28/04/2021 | Revisado: 05/05/2021 | Aceito: 10/05/2021 | Publicado: 26/05/2021

Rafaela Oliveira Brandão

ORCID: https://orcid.org/0000-0002-9640-5760 Centro Universitário Gama e Souza, Brasil E-mail: raphaela.brandao@hotmail.com

Camila Marques Costa

ORCID: https://orcid.org/0000-0002-8345-1047 Centro Universitário Gama e Souza, Brasil E-mail: camillamarques.ufrj@gmail.com

Estêvão Rios Monteiro

ORCID: https://orcid.org/0000-0003-1866-553X Centro Universitário Augusto Motta, Brasil Universidade Federal do Rio de Janeiro, Brasil E-mail: profestevaomonteiro@gmail.com

Victor Gonçalves Corrêa Neto ORCID: https://orcid.org/0000-0003-3133-1630 Centro Universitário Gama e Souza, Brasil Universidade Estácio de Sá, Brasil E-mail: victorgcn@hotmail.com

Felipe da Silva Triani

ORCID: https://orcid.org/0000-0001-6470-8823 Centro Universitário Gama e Souza, Brasil Universidade Estácio de Sá, Brasil E-mail: felipetriani@gmail.com

\begin{abstract}
Resumo
O objetivo do presente estudo foi analisar a associação entre o nível de atividade física e o comportamento sedentário de estudantes de Educação Física de uma faculdade da Zona Oeste do Estado do Rio de Janeiro. Para isso um grupo amostral com 90 estudantes, sendo 56 do sexo masculino e 34 do sexo feminino foi formado por conveniência. Todos os sujeitos responderam ao Questionário Internacional de Atividade Física e foram categorizados como satisfatoriamente ativos ou insatisfatoriamente ativos, bem como, tiveram o relato de seu tempo sentado quantificados representando o comportamento sedentário. Para avaliar a associação entre as distribuições se aplicou o teste do QuiQuadrado e foi aceito um nível de significância de $5 \%$ ( $\mathrm{p}<0,05)$. Não foi encontrada associação entre nível de atividade física e comportamento sedentário no grupo amostral $(\mathrm{p}=0,408)$. Conclui-se então, que indivíduos considerados satisfatoriamente ativos não estão necessariamente dentro dos critérios que hipoteticamente exercem proteção no que diz respeito à saúde se for levado em consideração o contraponto que o comportamento sedentário representa sobre tal tela. Ainda, a falta de associação aponta um caminho incongruente no que diz respeito as categorizações do questionário, retratando uma possível fragilidade desse instrumento.
\end{abstract}

Palavras-chave: Saúde; Saúde do estudante; Exercício; Sedentarismo.

\begin{abstract}
The aim of the present study was to analyze the association between the level of physical activity and the sedentary behavior of Physical Education students at a college in the West Zone of the State of Rio de Janeiro. For this, a sample group of 90 students, 56 male and 34 females, was formed for convenience. All subjects answered the International Physical Activity Questionnaire and were categorized as satisfactorily active or unsatisfactorily active, as well as having their sitting time quantified representing sedentary behavior. To assess the association between the distributions, the Chi-Square test was applied and a significance level of $5 \%(\mathrm{p}<0.05)$ was accepted. No association was found between the level of physical activity and sedentary behavior in the sample group $(p=0,408)$. It is concluded, then, that individuals considered satisfactorily active are not necessarily within the criteria that hypothetically exercise protection with regard to health if the counterpoint that sedentary behavior represents on such a screen is taken into account. Still, the lack of association points to an incongruous path with regard to the questionnaire's categorizations, portraying a possible weakness of this instrument.
\end{abstract}

Keywords: Health; Student health; Exercise; Sedentary lifestyle. 


\begin{abstract}
Resumen
El objetivo del presente estudio fue analizar la asociación entre el nivel de actividad física y el comportamiento sedentario de estudiantes de Educación Física de un colegio de la Zona Oeste del Estado de Rio de Janeiro. Para ello, se conformó por conveniencia un grupo de muestra de 90 estudiantes, 56 hombres y 34 mujeres. Todos los sujetos respondieron el Cuestionario Internacional de Actividad Física y fueron categorizados como satisfactoriamente activos o insatisfactoriamente activos, además de cuantificar su tiempo sentado representando un comportamiento sedentario. Para evaluar la asociación entre las distribuciones se aplicó la prueba de Chi-Cuadrado y se aceptó un nivel de significancia del 5\% (p <0.05). No se encontró asociación entre el nivel de actividad física y el comportamiento sedentario en el grupo de muestra $(\mathrm{p}=0,408)$. Se concluye, entonces, que los individuos considerados satisfactoriamente activos no necesariamente se encuentran dentro de los criterios que hipotéticamente ejercen protección en lo que respecta a la salud si se tiene en cuenta el contrapunto que representa el sedentarismo en dicha pantalla. Aún así, la falta de asociación apunta a un camino incongruente con respecto a las categorizaciones del cuestionario, retratando una posible debilidad de este instrumento.
\end{abstract}

Palabras clave: Salud; Salud del estudiante; Ejercicio; Sedentario.

\title{
1. Introdução
}

Nas últimas décadas, a população tornou-se cada vez menos ativa, com adultos gastando $70 \%$ ou mais de suas horas de vigília sentados, com isso, o dito comportamento sedentário habitual (muitas horas sentado), foi considerado um expressivo fator de risco cardiometabólico e de mortalidade por todas as causas, mesmo que paralelamente se realize atividades físicas de leves a extenuantes (Owen et al., 2010). Pesquisa recentemente publicada pelo Instituto Brasileiro de Geografia e Estatística (IBGE, 2017) apontou um alto índice de brasileiros que apresentam comportamento sedentário. Ainda, o inquérito apontou que 76\% dos brasileiros com idade de 15 anos ou mais não praticavam nenhum tipo de esporte. Dentre os principais motivos apresentados para tal comportamento foram relatados a falta de tempo, falta de interesse, problemas de saúde ou idade avançada. Essa grande prevalência de sujeitos sem envolvimento com práticas esportivas pode retratar um cenário em que a análise temporal corrobora congruentemente a mudanças ambientais com a redução dos níveis de atividade física.

Fazendo uma comparação com três ou quatro décadas atrás, nota-se que as pessoas têm se tornado cada vez menos ativas. As facilidades do mundo contemporâneo sustentadas pelas premissas do avanço tecnológico tornam o cotidiano mais prático, construindo um cenário adverso para rotinas mais ativas. Nos dias atuais, tudo está ao alcance das mãos, seja no trabalho ou em casa, fato que implica o aumento do comportamento dito como sedentário restringindo uma rotina que possivelmente seria subsidiada em atividades que aumentem o dispêndio energético, independentemente de serem exercícios estruturados, supervisionados e programados, atividades físicas de uma forma geral se caracterizam por contração muscular com aumento do dispêndio energético. Tais atividades poderiam ser tidas como precursoras de ambientes menos hostis a um comportamento menos caracterizado como sedentário (Caspersen; Powell; Christerson, 1985; Owen et al., 2017).

Diante do cenário posto em tela em que se pesem os fatores de risco para doenças crônico degenerativas, diversas variáveis devem ser levadas em consideração quando da reflexão sobre a gênese de diversas patologias. Destacando hábitos como tabagismo, etilismo e alimentação inadequada (Brasil, 2009), baixos níveis de atividade física também são sugeridos pela literatura como uma pontual variável independente no que diz respeito à fomentação de estados clínicos adversos (Ramsey et al., 2008), outrossim, refletindo antagonicamente, um maior nível de atividade física parece possivelmente despontar como um fator impactante positivamente na saúde da população. Estudos têm apontado que a prática regular de atividade física exerce efeito positivo sobre a capacidade cardiorrespiratória, aumento na expectativa de vida, aumento nos níveis de autoestima, da autoimagem e redução do estresse. Além disso, o discurso da saúde e da qualidade de vida divulgados massivamente pela mídia tem contribuído para o aumento da prevalência de indivíduos em busca de atividades físicas (Sabe, 2001), embora dados sobre o engajamento nas atividades não sejam muito otimistas.

O dito comportamento sedentário aparece como fator de risco prevalente para doenças cardiovasculares, obesidade, doença coronariana, hipertensão, diabete tipo 2 , osteoporose, câncer de cólon e depressão. Ainda, tampem aumenta o risco de 
condições como a obesidade que parece ser um fator de risco importante para injurias como a hipertensão arterial sistêmica (Katzmarzyk et al., 2004; Martins et al., 2010). O que ainda está incerto é o quanto um estilo de vida mais ativo pode estar associado a um menor comportamento sedentário, ou se ambos, nível de atividade física e hábitos sedentários coexistem de maneira independente, fomentando assim uma encruzilhada no que diz respeito as reflexões sobre atividade física e saúde.

Sendo assim, o presente trabalho teve como objetivo analisar a associação entre o nível de atividade física e o comportamento sedentário de estudantes de Educação Física de uma faculdade da Zona Oeste do Estado do Rio de Janeiro.

\section{Metodologia}

\subsection{Tipo de estudo}

O estudo em tela é uma pesquisa do tipo transversal de cunho observacional com característica quantitativa (Estrela, 2018).

\subsection{Amostra}

A amostra foi composta por 90 estudantes, sendo 56 do sexo masculino e 34 do sexo feminino, todos estudantes da mesma unidade de uma instituição de ensino superior privada localizada na zona oeste do Rio de Janeiro. A composição do grupo amostral aconteceu por conveniência, sendo todos os sujeitos estudantes do curso de Educação Física.

Todos os indivíduos eram maiores de idade, foram voluntários e esclarecidos a respeito de todos os procedimentos da rotina de coleta de dados, bem como assinaram o termo de consentimento livre e esclarecido (TCLE).

A presente pesquisa foi aprovada pelo Comitê de Ética em Pesquisa da Universidade do Grande Rio, sob a guarda do CAAE de número 61550916.1.0000.5283.

\subsection{Instrumento}

Para avaliar o nível de atividade física foi utilizado o questionário internacional de atividade física (IPAQ) versão curta. Esse instrumento já teve sua reprodutibilidade testada em amostra composta por ambos os sexos obtendo resultados estatísticos satisfatórios (Matsudo, 2010).

\subsection{Procedimentos}

Os alunos do curso de Educação Física da instituição de ensino eram abordados dentro da instituição para participação no estudo. Todo o objetivo da pesquisa, bem como o procedimento era explicado e após a aceitação e a concordância por escrito no TCLE o questionário IPAQ era entregue ao sujeito que era encaminhado para uma sala tranquila onde com a presença de um pesquisador para eventuais dúvidas, preenchia o instrumento.

Após a tabulação das respostas, os indivíduos foram descritivamente classificados dentro das categorizações propostas pelo IPAQ: sedentário, irregularmente ativo B, irregularmente ativo A, ativo e muito ativo. No entanto, para efeito de tratamento foram considerados como satisfatoriamente ativos os indivíduos alocados nas categorias "ativo" e "muito ativo", bem como foram considerados insatisfatoriamente ativos os sujeitos que foram categorizados como "irregularmente ativo A", "irregularmente ativo B" e "sedentários", conforme a categorização sugerida para o IPAQ. As horas sentado durante os dias de semana ou fins de semana também foram registradas e quantificadas em uma única categoria que foi fracionada em "mais de 5 horas sentado" ou "menos de 5 horas sentado", o ponto de corte foi estabelecido tendo como base a variabilidade dessa medida no grupo amostral calculada pela amplitude total. 


\subsection{Tratamento Estatístico}

A estatística descritiva foi aplicada em valores percentuais para descrever a prevalência dos indivíduos de acordo com seu nível de atividade física. Tendo em vista a característica categórica dos dados em análise as inferências foram feitas por teste não paramétrico. O qui-quadrado foi aplicado para mensurar as associações entre as distribuições. Para todo tratamento inferencial foi aceito um nível de significância de $5 \%(\mathrm{p}<0,05)$. Foi utilizado para a análise estatística o Statistical Package for the Social Sciences (SPSS), versão 20.0.

\section{Resultados e Discussão}

Quanto à prevalência dos sujeitos quanto ao nível de atividade física $51,11 \%$ dos indivíduos se situaram nas estratificações que compõe o grupo "satisfatoriamente ativos", enquanto $48,89 \%$ dos sujeitos foram classificados no estrato “insatisfatoriamente ativos". A prevalência do grupo amostral para cada categorização do IPAQ está ilustrada na Tabela 1.

Tabela 1. Prevalência para cada categorização de nível de atividade física segundo o IPAQ

\begin{tabular}{ll}
\hline Nível de atividade física & Prevalência (\%) \\
\hline Muito ativo & 20 \\
Ativo & 31,11 \\
Irregularmente ativo A & 36,66 \\
Irregularmente ativo B & 8,9 \\
Sedentário & 3,33 \\
\hline
\end{tabular}

Fonte: Autores.

A análise inferencial não foi capaz de identificar associação significativa ( $\mathrm{p}=0,408)$ entre o nível de atividade física e o tempo de horas sentados do grupo amostral em análise (Tabela 2).

Tabela 2. Tabela de contingência das distribuições de horas sentado por nível de atividade física.

\begin{tabular}{llllllll}
\hline & \multicolumn{2}{c}{$>$} & & $<5$ h sentado & Total & $P$ \\
\hline & $\mathrm{n}$ & $\%$ & $\mathrm{n}$ & $\%$ & $\mathrm{n}$ & $\%$ & \\
\hline Satisfatoriamente ativos & 19 & 46,34 & 27 & 55,10 & 46 & 51,11 & 0,408 \\
Insatisfatoriamente ativos & 22 & 53,66 & 22 & 44,90 & 44 & 48,89 & \\
\hline Total & 41 & 100 & 49 & 100 & 90 & 100 \\
\hline
\end{tabular}

Fonte: Autores.

O presente estudo buscou analisar a associação entre o nível de atividade física e o comportamento sedentário de estudantes de Educação Física de uma Faculdade da Zona Oeste do Rio de Janeiro. Os resultados obtidos revelam que dos 46 estudantes classificados como satisfatoriamente ativos, 19 passam mais de 5 horas do dia em comportamento sedentário e dos 44 classificados como insatisfatoriamente ativos, metade apresentou comportamento sedentário.

Inicialmente é importante perceber que 49\% dos estudantes foram classificados como insatisfatoriamente ativos, evidenciando uma contrariedade a estudos anteriores (Silva et al., 1997; Bielemann et al., 2007) que identificaram uma alta prevalência das categorizações ativos e muito ativos do IPAQ, categorizações essas, que denotam na presente investigação o grupo satisfatoriamente ativo. Dessa maneira, ser estudante de Educação Física e ser ativo fisicamente são atributos que não 
devem ser vistos de forma congruente via de regra. Diante de tal tela, cabe pontuar que considerar tal grupo como ativos arbitrariamente pode simplesmente ser a tradução de um constructo paradigmático.

Adicionalmente, no que se refere à atividade física, há duas recomendações que perpassam o universo de discussões no campo da Educação Física, a primeira apontada pelo Center for Disease Control - American College of Sports Medicine (CDC/ACSM) "que todo indivíduo deve acumular ao menos 30 minutos de atividade física, na maioria dos dias da semana, em intensidade moderada, de forma contínua ou acumulada" (Matsudo; Matsudo; Araújo, 2002). No entanto, embora essa recomendação esteja presente há mais de dez anos no campo da Educação Física, se o conceito de comportamento sedentário como fator de risco para a saúde for inserido como um contraponto há de se perceber que essa recomendação não protege da exposição aos riscos cardiometabólicos e mortalidade por todas as causas (Owen et al., 2010).

Nessa mesma perspectiva, Donnelly et al. (2009) recomendam a prática da atividade física entre 150 e 200 minutos por semana. Porém, adotar a prática de atividade física durante esse período recomendado sem manter seu estilo de vida ativo, também não protege o sujeito aos riscos associados ao comportamento sedentário. Dessa maneira, ser satisfatoriamente ativo não é condição inversamente proporcional ao comportamento sedentário, pois considerando os resultados da investigação em tela, há possibilidade de pessoas muito ativas apresentarem comportamento sedentário.

Por fim, cabe pormenorizar o quanto é problemático discutir os conceitos atrelados ao termo comportamento sedentário, ou mesmo o de sedentário, que emerge com caráter não científico na forma em que é empregada na maioria das vezes (Palma; Vilaça; Assis, 2014). A dicotomia do questionário utilizado na investigação em tela já foi posta em pauta sobre o prisma que emerge na fragilidade de utilizar medidas que mensuram nível de atividade física e comportamento sedentário no mesmo instrumento (Corrêa Neto; Palma, 2014). Os achados aqui descritos retratam a falta de associação entre essas questões corroborando a incongruência das informações levantadas. Ainda, o viés de memória que pode acontecer em relação a instrumentos do tipo self report também deve ser levado em consideração no que diz respeito à fragilidade da categorização dos indivíduos com esse tipo de estratégia (Medronho et al., 2009).

Explorando o âmbito de fragilidades das medidas de atividade física, cabe destacar ainda o possível caráter mítico que alicerça um discurso hegemônico sobre as associações entre atividade física e saúde. Associações, mesmo quando existem são estratégias matemáticas para estimação de riscos ou chances, enquanto a visão de causalidade discursada entre essas variáveis pressupõe o acordo de diversos critérios que muitas vezes não são analisados. Ainda, o discurso de causa e efeito deve atender o critério sine quo non de temporalidade, onde exposição precede o desfecho (Medronho et al., 2009), essa ordem temporal não pode ser observada em estudos transversais que muitas vezes por sua menor dificuldade de operacionalização são utilizados como égide desse prisma. Mesmo uma forte associação sendo um critério a ser levado em consideração para causalidade, um bloco de evidências não retrata associação significativa entre atividade física e saúde (Yoshinaga et al., 2011; Gaya et al., 2011; Aounallah-Skhiri et al. 2012).

Cabe ainda pontuar que considerando que a classificação do IPAQ leva em conta apenas a prática de atividade física, o mesmo deveria ser objeto de reflexão quando da sua aplicação pensando na associação com gêneses patológicas, tendo em vista que sujeitos satisfatoriamente ativos podem denotar um grande tempo em comportamento sedentário, e que o caráter dicotômico dos resultados corroborados pela falta de associação estatística entre as questões abordadas, podem eleger grupos sem comparabilidade confundindo discussões em tal âmbito. A utilização de alguma estratégia que pudesse inserir de alguma forma as horas sentados na classificação por categorização do IPAQ poderia ser uma tentativa de normalizar a classificação levando em consideração tanto as características da prática das atividades físicas quanto do comportamento sedentário.

Nesse sentido, vale a pena refletir sobre o discurso inferido a essas relações tanto no âmbito mítico e hegemônico, quanto na fragilidade dos instrumentos e dos desenhos de estudos empregados para alicerçar a popularidade de um diálogo 
unilateral que pode estar sendo construído sobre uma premissa de pânico moral e culpabilização desconsiderando uma possível vulnerabilidade que transpassa o individual (Palma et al., 2003).

O presente estudo apresenta algumas limitações, a maior delas possivelmente o caráter de conveniência de seleção da amostra. Porém, dentre as características avaliadas o único resultado que parece ser alvo de comprometimento seria a prevalência de sujeitos nas categorizações satisfatoriamente ativos e insatisfatoriamente ativos, já que grupos populacionais podem apresentar características bem diferentes no que diz respeito a pratica de atividades físico - esportivas, porém, a principal informação aqui levantada e discutida não diz respeito à prevalência de indivíduos mais ou menos ativos, e sim a associação entre nível de atividade física e comportamento sedentário, e na análise desse tipo de associação não parece a forma de seleção de a amostra ter poder de influenciar os resultados. Ainda, o desenho do tipo transversal não permite discursos sobre causalidade (Medronho et al., 2009), mas cabe ressaltar que o objetivo do presente trabalho não circunda em pretensão de discursar causa e efeito e sim de estabelecer fragilidades em duas medidas que se mostram de encontro entre elas e que costumeiramente são discursadas como em comum.

Cabe destacar que a presente investigação é a primeira que se tem conhecimento que abordou a associação entre nível de atividade física e comportamento sedentário levando em consideração o mesmo instrumento, e isso, corrobora a relevância do estudo que põe em pauta tanto a falta da de relação entre as informações coletadas, quanto a uma importante reflexão sore o paradigma de que determinado ponto de corte relacionado ao nível de atividade física pode exercer um efeito protetor sore a saúde da população, desconsiderando o risco que atividades tidas como de características sedentárias podem representar em tal cenário.

\section{Considerações Finais}

Não foi identificada associação significativa entre o nível de atividade física e o tempo de horas sentado. Tal achado nos leva pontualmente a duas conclusões, a primeira que estudantes de Educação Física mesmo que com satisfatórios níveis de atividade física, podem passar grande tempo do seu dia em atitudes de comportamento sedentário, o que talvez independente da prática esportiva os mantivessem em uma zona de risco para desenvolvimento de doenças crônico degenerativas. A segunda guisa de conclusão diz respeito à análise crítica do instrumento utilizado que mostra resultados dicotômicos entre variáveis hipoteticamente associadas, inserindo um possível viés de informação nas categorizações sugeridas pelo IPAQ quando o mesmo é classificado tendo como base unicamente as práticas físicas e desprezando o tempo de horas sentado nas categorizações como de fato tem sido descrito na literatura.

\section{Agradecimentos}

Os autores agradecem a participação de todos os voluntários dessa pesquisa.

\section{Referências}

Aounallah-Skhiri, H., El Ati, J., Traissac, P., Ben Romdhane, H., Eymard-Duvernay, S. Delpeuch, F., Achour, N., \& Maire, B. (2012). Blood pressure and associated factors in a north African adolescent population. A national cross-sectional study in Tunisia. BMC Public Health, 12 (1), 98.

Bielemann, R., Karini, G., Azevedo, M. R., \& Reichert, F. F. (2007). Prática de atividade física no lazer entre acadêmicos de Educação Física e fatores associados. Revista Brasileira de Atividade Física e Saúde, 12 (3), 65-72.

Brasil. (2009). Estatística e informação em saúde. Ministério da Saúde.

Corrêa Neto, V., \& Palma, A. (2014). Pressão arterial e suas associações com atividade física e obesidade em adolescentes: uma revisão sistemática. Ciência e Saúde Coletiva, 19 (3), 797-818.

IBGE. (2017). Falta de tempo e de interesse são os principais motivos para não se praticar esportes no Brasil. http://saladeimprensa.ibge.gov.br/noticias?view=noticia\&id=1\&busca=1\&idnoticia=3432 
Caspersen, C. J., Powell, K. E., \& Christerson, G. M. (1985). Physical Activity, Exercise, and Physical Fitness: Definitions and Distinctions for Health-Related Research. Public Health Reports, 100 (2), 126-131.

Donnelly, J. E., Blair, S. N., Jakicic, J. M., Manore, M. M., Rankin, J. W., \& Smith B. K. (2009). American College of Sports Medicine. American College of Sports Medicine Position Stand. Appropriate physical activity intervention strategies for weight loss and prevention of weight regain for adults. Med Sci Sports Exerc, 41 (2), 459-471.

Estrela, C. (2018). Metodologia Científica: Ciência, Ensino, Pesquisa. Editora Artes Médicas

Gaya, A. R., Silva, P., Martins, C., Gaya, A., Ribeiro, J. C., \& Mota, J. (2011). Association of leisure time physical activity and sports competition activities with high blood pressure levels: study carried out in a sample of Portuguese children and adolescents. Child Care Health Dev, 37 (3), 329-334.

Katzmarzyk, P. T., Srinivasan, S. R., Chen, W., Malina, R. M., Bouchard, C., \& Berenson, G. S. (2004). Body mass index, waist circumference, and clustering of cardiovascular risk factors in a biracial sample of children and adolescents. Pediatrics, 114 (2), 198-205.

Martins, M. C. C., Ricarte, I. F., Rocha, C. H. L., Maia, R. B., Silva, V. B., \& Veras, A. B. (2010). Pressão arterial, excesso de peso e nível de atividade física em estudantes de universidade pública. Arquivos Brasileiros de Cardiologia, 95 (2), 192-19.

Matsudo, S., Aráujo, T., Matsudo, V., Andrade, D., Andrade, E., Oliveira, L. C., \& Braggion, C. (2001). Questionário internacional de atividade física (IPAQ): estudo de validade e reprodutibilidade no Brasil. Revista Brasileira de Atividade Física e Saúde, 6 (1), 5-18.

Matsudo, S. S. M., Matsudo, V. K. R., \& Araújo, T. (2002). Nível de atividade física da população do Estado de São Paulo: Análise de acordo com o gênero, idade, nível sócio-econômico, distribuição geográfica e de conhecimento. Revista Brasileira de Ciência e Movimento, 10 (1), 41-50.

Medronho, R. M., Bloch, K. V., Luiz, R R., \& Werneck, G. L. (2009). Epidemiologia. (2a ed.), Atheneu.

Owen, N., Sparling, P. B., Healy, G. V. N., Dunstan, D. W., \& Mathews, C. E. (2010). Sedentary behavior: emerging evidence for a new health risk. Mayo Clinic Proceedings, 85 (12), 1138-1141.

Owen, N., Phillip, B., Genevieve, N., Healy, D., Dunstan, W., \& Matthews, C. E. (2017). Sedentary Behavior: Emerging Evidence for a New Health Risk. Mayo Clinic Proceedings, 95 (1), 1138-1141.

Palma, A., Salomão, L. C., Nicolodi, A. A. G., \& Caldas, A. (2003). Reflexões acerca da adesão aos exercícios físicos: comportamento de risco ou vulnerabilidade? Movimento, 9 (3), 83-100.

Palma, A., Vilaça, M. M., \& Assis, M. R. (2014). Excertos sobre o sedentarismo. Revista Brasileira de Ciência do Esporte. 36 (3), 656-662.

Ramsey, F., Ussery-hall, A., Garcia, D., Mcdonald, G., Easton, A., Kambon, M., Balluz, L., Garvin, W., \& Vigent, J. (2008). Prevalence of selected risk behaviors and chronic diseases - Behavioral Risk Factor Surveillance System (BRFSS), 39 steps communities, United States, 2005. Surveillance Summaries, $57(1), 1-20$.

Saba, F. 2001. Aderência: a prática do exercício físico em academias. Manole.

Silva, G., Rosa, R., Rosa, M., Melo, C., Miranda, R., \& Filho M. (1997). Avaliação do nível de atividade física de estudantes de graduação das áreas saúde/biológica. Revista Brasileira de Medicina do Esporte, 13 (11), 39-42.

Yoshinaga, M., Hatake, S., Tachikawa, T., Shinomiya, M., Miyazaki, A., \& Takahashi, H. (2011). Impact of lifestyles of adolescents and their parents on cardiovascular risk factors in adolescents. J Atheros Thromb, 18 (11), 981-990. 